\title{
Analysis Of Distance-Based Mental Health Support For Underrepresented University Students
}

\author{
Lucretia Williams* \\ University of California, Irvine, USA \\ Rebecca Black $^{*}$
University of California, Irvine, USA
}

\author{
Candace Williams \\ University of California, Irvine, USA \\ Gillian R. Hayes
University of California, Irvine, USA
}

Gloria Washington*

Howard University, USA

\author{
Michael Allotey \\ University of California, Irvine, USA \\ Leah Clements \\ Howard University, USA
}

\begin{abstract}
Recent reports have shown a growing demand for mental health resources and services on university campuses for Black and Latinx students. These students have a higher rate of unmet mental health needs and are more likely to experience mental health problems. Offering a technical solution is promising for navigating on campus mental health services. In this paper, we present findings from a preliminary study focused on understanding the mental health related technology practices and preferences of university students and a content analysis of 60 U.S. college and university counseling center websites. Findings highlight how university students' desire for applications that integrate with their existing on-campus offerings contrasted with the apparent offerings of campus counseling centers.
\end{abstract}

\section{CCS CONCEPTS}

- Human-centered computing; • Human-computer interaction (HCI). Additional KeyWords and Phrases: University Students, Mental Health, Telehealth, Systems;

\section{ACM Reference Format:}

Lucretia Williams, Candace Williams, Michael Allotey, Rebecca Black, Gillian R. Hayes, Leah Clements, and Gloria Washington. 2021. Analysis Of Distance-Based Mental Health Support For Underrepresented University Students. In CHI Conference on Human Factors in Computing Systems Extended Abstracts (CHI '21 Extended Abstracts), May 08-13, 2021, Yokohama, Japan. ACM, New York, NY, USA, 6 pages. https://doi.org/10.1145/3411763. 3451708

\section{INTRODUCTION}

Black and Latinx university students with mental health needs can experience greater challenges succeeding in higher education due to stigma within their communities $[6,7,18,20,22]$, bias in mental health care assessment [34], and difficulty with seeking and obtaining on-campus mental health resources [43]. Highly diverse

${ }^{*}$ These authors contributed equally to this research.

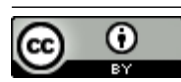

This work is licensed under a Creative Commons Attribution International 4.0 License.

CHI '21 Extended Abstracts, May 08-13, 2021, Yokohama, Japan

(C) 2021 Copyright held by the owner/author(s).

ACM ISBN 978-1-4503-8095-9/21/05

https://doi.org/10.1145/3411763.3451708 student populations living, learning, and working within the same university can lead to cultural barriers, feelings of isolation, and possible lack of representation for Black and Latinx students [9]. Current reports of police brutality $[1,3]$ and disproportionate rates of COVID-19 mortality in Black and Latinx populations [39, 41] have intensified mental well-being issues among these students. A variety of factors contribute to difficulty in seeking help, including the praise of resiliency [25] and interpretations of therapy or counseling as weakness [20].

In recent years, it has become more evident that these communities can benefit substantially from therapy and counseling [29,33] because of the extreme oppression that they have overcome. With Black and Latinx communities experiencing the long lasting effects of systemic racism in America, university students who belong to these respective communities are searching for a positive and supportive environment $[42,43]$ to address their mental health challenges.

University campuses can become a safe haven for Black and Latinx students when they leave home to pursue higher education; however, within their respective institutions, it is important that they feel supported and have easy access to resources that will help them through various cultural barriers. These resources should feel inclusive, accessible, and empowering. This will allow them to easily navigate spaces on campus to find the resources they feel will suit their needs. On-campus resources can better meet the needs of these students if school-wide systems are made more accessible, more culturally aware and sensitive, and further encourage these groups of students to visit specific on campus resources that meet their needs. Without such resources, Black and Latinx students are at substantial disadvantage [4].

Digital mental health interventions have been used to create online communities and resources for university students [42] in the form of web and mobile applications [36]. In this study, we present findings from an exploratory survey study that conveyed how Black and Latinx undergraduate and graduate students perceived the effectiveness of on campus counseling services and provided insight on tech solutions that can possibly close the accessibility gap. The goal of this survey study was to understand their experiences, detail their current resources, brainstorm potential technical support systems and evaluate the current systems. We also present findings from a content analysis of campus counseling center websites from the top 20 Predominately White Institutions (PWI), Hispanic 
Serving Institutions (HSI) and Historically Black Colleges and Universities (HBCU). The goal of the content analysis is to evaluate how current university systems are providing inclusive mental health resources to their students.

Findings from the survey study show that students struggle with navigating on-campus mental health resources and do not want another tech solution to solve the problem but instead want the existing systems fixed. These findings highlight how university students' desire for applications that integrate with their existing on-campus offerings contrast with the apparent offerings of campus counseling centers or the broader mental health tech ecosystem. Students and universities alike demonstrate through our survey responses and content analysis an interest in greater access, a focus on wellness, peer-community support, and culturally responsive mental health options. By considering mental health, and the technologies that support mental health, as an enterprise-wide issue and a comprehensive user experience challenge, we can see how such technologies might be designed in a cohesive manner to support student success broadly.

In this paper, we discuss considerations for using technology to augment existing systems within and surrounding counseling centers on university campuses for researchers, faculty, and university staff seeking to improve access to healthcare through technology. The main contributions of this work are: (1) Identifying specific mental health resources Black and Latinx university students seek for on their respective campuses and (2) Design implications for creating more efficient and effective systems for on campus counseling services.

\section{RELATED WORK}

This paper adds to a greater body of HCI research around digital mental health technology. In this section, we will explore works related to the mental health of Black and Latinx students, and articulate how our research expands on this literature.

\subsection{Mental Health Technology Interventions For University Students}

Technology interventions to support the mental health of university students are of increasing interest to the $\mathrm{HCI}$ community. $\mathrm{HCI}$ researchers have been developing solutions to support the mental health of university students through ubiquitous applications and online support. University students experience a lifestyle that places them under high amounts of stress when they are often living miles away from family, friends, and other traditional support structures. Due to cultural stigmas [7], students from some marginalized groups are often reluctant to seek out in-person mental health support. Thus, digital mental health interventions can offer promising options for individuals from these groups within higher education [43].

A variety of mHealth applications have been provided as supportive solutions to help close the gap between balancing current lifestyle and seeking help in multiple forms [8]. Solutions such as mindfulness-based text messages [19,21], mobile apps [10, 36], web apps [35], wearable devices [2,13], and online support groups $[27,28]$ have been studied and developed by researchers within the HCI community. Medical studies have also been exploring the use of technology to promote mental health awareness. A study in Frontiers of Psychiatry identifies the issues and benefits that personal computing systems can offer university students for mental health [17]. Researchers in this domain highlight how mental health services supported by technology need to be optimized for mobile usability, and how consideration should be given to the use of native apps and web apps. This study identifies a large gap of research-to-practice implementation, emphasizing how very few studies actually focus on implementing technology-enabled mental health resources. Our work contributes to the larger conversation related to implementation of technology support for mental health services for university students on campus by offering concrete design suggestions that are grounded in student voices.

Following a series of design workshops with university students and counseling center staff, researchers identified students' need for social environments and support networks [16]. The results of that work illustrate the influential position of well-known peers and their supportive positions in shaping the types of digital mental health resources students want and how they can learn about mental health resources. Our work is similar in its findings of student preferences and using technology as a solution to give students what they prefer but this work takes a slight turn in analyzing the main technology that is currently being used for students on campus.

Our work contributes to understanding the perspectives university students have on using technology to support their mental health but focuses solely on the perspectives of minority students. It adds to the large body of exploratory research used to develop supportive mental health tech solutions for university students.

\section{METHODS}

After considering the results of the survey, questions began to arise about the systems and processes of university counseling centers for the kinds of approaches that our data suggested were needed. This prompted the research team to conduct a content analysis of 60 counseling websites from prominent U.S. colleges and universities [24]. In this section, we describe each phase of this project.

\subsection{Exploratory Survey}

The exploratory survey study was conducted with the dual purpose of gathering insights into how Black and Latinx students navigate existing on-campus counseling resources, and eliciting student input on how technology might be used to improve access to these resources. The survey was sent out electronically by the office of access and inclusion for two schools at the lead author's university through a listserv that consisted of university students in multiple higher educational programs (Appendix B). A total of 36 university students ( 25 undergradu- ate and 11 graduate) who self- identified as Black and Latinx participated in the survey study. All participants are currently enrolled in a degree program. The survey was anonymous and did not contain any identifying information (i.e. name, school, major). The survey was hosted on Qualtrics survey platform with a total of 20 questions in both long and short answer format. The researchers conducted a qualitative analysis of two short answer survey responses. First-cycle coding [32] involved an inductive approach in which descriptive and in- 
vivo codes were derived from participant responses. Second cycle coding [32] focused on identifying thematic patterns in the data and ways in which participants' responses converged and diverged thematically. Qualtrics software was used for statistical analysis of multiple choice questions.

\subsection{Content Analysis}

Three researchers, the first and last two authors, conducted a content analysis of 60 U.S. college and university counseling center websites [24]. Sites analyzed included the 20 top- ranked each of Predominantly White Institutions, Hispanic Serving Institutions, and Historically Black Colleges and Universities (as ranked by US News and World Report in August 2020) for a total of 60 websites. One researcher did a google search on "college/university name" + counseling center to find the counseling website of each specific school and then navigated to the highest level web page for the counseling center. The researchers identified the initial units and categories of analysis based on a combination of their collective knowledge of mental health resources and the thematic categories identified through coding the student survey responses. Using this initial code book of 18 codes, all three researchers coded a subset of 6 sites. The team then met to compare their results, discuss the coding process, and identify codes that needed to be clarified, expanded, collapsed, added, or deleted. Based on that discussion, the researchers arrived at the final operational definitions for 16 codes (Appendix A). The researchers then added an additional six sites and re-coded the first six, for a total of $12(20 \%$ of the total sites to be coded) with an inter rater reliability of .86 (93.06\% agreement). Finally, each researcher independently coded an additional 16 sites for a total of 60 sites.

\section{FINDINGS}

Similarly to previous research detailing the experiences of university students' mental health [16], survey respondents described barriers to accessing mental health care, such as access to resources and tools to help them cope. The findings showed no difference between the type of school the students attended and student mental health care preferences. Students emphasized wanting to utilize peer support groups and local community resources, and wishing for more efficient access to on-campus resources with scheduling appointments and finding counselors that best suited their needs. Notably, this project began with the intent to engage heavily in mobile mental health support. However, these findings clearly indicate the need to redesign and develop improved fundamental technical infrastructure around mental health on college campuses. In this section, we highlight areas of improving access, holistic approach, community and peer support, and culturally responsive options.

\subsection{Improving Access}

Easy access to appropriate mental health services on campus is essential to helping students seek out and utilize the services intended for them. The notion of access was present in the data in two distinct ways, which we describe as improving navigational access (the ability to clearly navigate resources) and situational access (the ability to access resources by a variety of methods to suit the needs of a variety of situations).
In terms of improving navigational access, survey participants uniformly expressed difficulty with finding resources and had limited knowledge of what resources and services were available to them on campus. Students expressed strong preferences for wanting better access to services on campus. For example, one participant identified wanting "easier access to mental health professionals," referring specifically to the difficulty of actually making contact with a counselor. Other students reported wanting easier access to resources. This lack of navigational access was also evident to the researchers as they coded the 60 counseling center websites. Many resources were difficult to find because they were housed in non-intuitive sections (e.g., diversity-related resources were almost exclusively housed under group therapy), or required clicking through multiple pages, tabs, and/or links to locate. Taken together, these findings suggest that mental health resources and services are often not presented in an intuitive fashion, which is a significant shortcoming when the students attempting to navigate them may already be experiencing high levels of stress or mental distress.

Given the stigma surrounding seeking help with mental health for Black and Latinx students, it may be a challenge for these students to inquire about services in person due to the fear of being seen by fellow peers or faculty. Therefore, it was not surprising that study participants also expressed a strong desire for what we call situational access, or access to a variety of means of accessing different mental health resources that were suited to their current situation or needs. For example, suggestions included online appointment scheduling, an event calendar for online group discussions, and anonymous chat box discussions.

This need for situational access was also reflected in the content analysis. Despite the fact that COVID-19 has pushed more U.S. colleges and universities to offer telehealth services than ever before, our analysis revealed that even post-pandemic, many institutions were sorely lacking in online resources. Of the 60 sites reviewed, ten offered scheduled counseling via phone, and five offered counseling via video, 34 offered both, and 11 offered no remote options or were unclear in their offerings. Immediate counseling were offered via phone or video at 39 schools, with two of those supplementing with chat-based immediate intervention. Given these limited options, particularly during remote educational terms, the limits of access for students is substantial. However, it is worth noting that expanded telehealth options alone is not a solution to the issue of increased access. Students living in dorms or shared apartments frequently struggle to find a quiet space in which they can also talk about private concerns. Even making the phone call to book an appointment can potentially be a challenge, but only 12 sites offered online appointment booking and only 23 offered an online health assessment prior to booking an appointment. Even those schools that advertised immediate chat sessions with counselors had no easily discernible way to access these chats. This lack of both navigational and situational access presents significant opportunity for technology to relieve the burden of students seeking services by providing an easily navigable, intuitive, and user-focused interface, and a broader range of resources that can be accessed discreetly, independently, and remotely. Finally, it is unclear whether these universities will maintain what telehealth offerings and external online resources they do offer following the pandemic. 


\subsection{A Holistic Approach To Mental Health}

University students are often under high amounts of stress, with busy schedules and heavy work- loads. Managing these demands while maintaining mental health can be challenging [5, 14, 29]. Our findings demonstrate that of the multiple resources presented to students on campus (e.g., psychiatric services, individual counseling, etc.), students were most interested in having more resources focused on addressing their health care needs holistically. Wellness, for example, was a central focus of concern for students. For example, student survey respondents requested "healthy tips to deal with different types of mental health issues", "mood tracking," and "healthy exercises for mental health improvement, meditation/calming practices." These approaches were well-represented in counseling center websites, with 53 out of 60 (88\%) offering some form of wellnessrelated resources to their students, and $41(68 \%)$ were coded as having a wellness ethos as opposed to a more traditional medical model [15] pervading the entire site.

Students were specific in their descriptions of the types of resources that they believed could contribute to their overall wellbeing, such as, "Memes, something to make you laugh; a step by step process of how to meditate and evaluate ourselves and mental state." They also requested wellness-focused "Therapy sessions, anonymous chats, reminders to do small self-care tasks." The websites themselves offered a variety of resources focusing on the whole student, such as study skills and time management workshops, yoga and meditation classes, and wellness apps, to name just a few. While our analysis revealed great interest in self-care and self-evaluation options to support students' mental health, and indeed many sites offered such self-care resources, however one challenge for both the students hoping to consume these resources and the counseling centers offering them is the limited efficacy study of such mHealth resources. Along these lines, in our content analysis, we noted that universities would limit their own recommendations by offering a disclaimer that distanced themselves from the external wellness and self-help resources that were included on the website. University students, already feeling stressed and a bit lost, are unlikely to be able to discern on their own the efficacy of any particular approach outside of professional care, and indeed, one respondent decried "not another app."

\subsection{Community and Peer Support}

Having a sense of community and belonging in an environment away from home plays a crucial role in a university student's experience [12, 26, 38], even more so for minoritized students [9, 11, 37]. When mental health challenges arise, students often want to be a part of a community that will help them cope and work through issues and that can commiserate and share similar experiences. University students find these communities in on-campus clubs and organizations, as well as the broader community, such as local churches and meet-up groups. Participants expressed a desire for local community resources and counselors that were external to campus, such as "How to find local black counselors and professionals, resources for local groups, self-care tips, hotline numbers, 24/7 communication line." Notably, in this quote, the respondent describes not only the types of resources needed but also hints at the need for cultural competency by explicitly calling out the need for "black counselors and professionals", a topic we take up in the next section.

In contrast to students' expressed desire for community-based forms of support, our content analysis revealed that only 39 of the 60 sites analyzed provided such resources. Additionally, even when provided, none of these resources connected directly into online offerings from those groups, provided mapping directly to them, or included any other integrated services students have come to expect from a modern customer-centric web experience.

Students also expressed wanting to have fellowship and the opportunity to engage in conversations with peers who are experiencing similar issues as them. Specifically, they asked for things like "Peer-to-peer conversations, alumni network, resources" and "A community of people that are dealing with similar issues." In addition, students provided a variety of options of peer support, including " $A$ chat box for anonymous discussions," event calendars, and distancebased peer mentoring. While our content analysis revealed that many institutions provided training and support for peer counseling services, none appeared to incorporate alumni as resources. In addition, it is worth noting that on the counseling websites, peer support was sometimes positioned as an outward-facing activity. For example, one university website framed peer support as referring a friend to the counseling center.

Please let us know if you are worried about a friend. We are also available to consult with faculty and staff about concerns they may have about their students. Give our office a call, or reach out to our Campus Awareness Response and Education (CARE) Team.

Another site positioned peer support as a limited resource that students should approach with caution.

Involve yourself only as far as you are willing to go. At times, in an attempt to reach or help a troubled friend, you may become more involved than time or skill permits. It is important to know the boundaries and limitations of your intervention.

However, these notions of peer support, while important, are not well-aligned with the fellowship and community-focused notions of peer support that students in our study expressed wanting. In addition, it is important to note that even when institutions offer peer counseling, it can be a significant challenge for minoritized students to find peers through such services in primarily white institutions. Some institutions addressed this need for community and shared- experience through group counseling and the creation of discussion groups focused on shared student backgrounds and identities (26 of 60). Nonetheless, there are clear discrepancies between what students in our study expressed a desire for, and what these institutions offer in terms of community and peer support.

\subsection{Culturally Responsive Mental Health Options}

Culturally responsive mental health resources minimize inequalities in mental health outcomes and access to services [31, 40]. Resources and activities culturally related to these students helps them build a sense of belonging and a broader community [23, 30]. Students care about the identities held by their counselors, and expressed 
wanting to learn and/or know more about them before scheduling an appointment. Student survey respondents expressed having access to counselors who they can relate to in culture and identity (i.e. ethnicity, gender, and sexual orientation). Alternatively, student survey respondents expressed wanting access to counselors that they can relate to. To illustrate, one participant explained that they would like to see which therapists on their campus identify themselves as a person of color, queer, or both, while another expressed a desire specifically for access to Black mental health professionals. Similarly, a different student asked for photos of nearby professionals wanting, "names and number of nearby professionals with pictures."

Despite students' clear preferences for finding mental health professionals with whom they feel some form of connection or affiliation, our content analysis revealed that finding this information and making these connections was not well-supported. 18 sites (30\%) had no bio information for counselors at all. For others, the information was often buried or difficult to find. Specifically, they were often text-only (i.e., no photos), contained minimal information, and/or only included name and professional degree.

There were, however, some clear exceptions, where bios were more expansive and included the names, photos, and specializations of the mental health professionals. The results indicate student preference for transparency of counselor's identity and specialty.

\section{LIMITATIONS AND FUTURE WORK}

While attempting to recruit survey participants from a variety of U.S. colleges and universities, the results of our exploratory survey are not fully representative of the mental health needs and preferences of Black and Latinx students on a variety of campuses. Some results may be biased by having the majority of the survey participants attend one specific school environment (e.g., PWI or HBCU). We did not specifically recruit student participants who have known mental health challenges that would specifically be interested in this study. Our content analysis of the 60 U.S. colleges and universities are confined to schools that are ranked top 20 in their respective category (PWI, HSI, and HBCU) U.S. News and World Report in August 2020 [24]. The interpretations of these findings is not a generalization of all college and university environments. Future work should include interviews and focus groups of Black and Latinx students who are interested in benefiting from better access to mental health services on campus as well as both a broader and deeper engagement with analysis of existing systems.

\section{CONCLUSION}

This study examines the mental health needs of Black and Latinx university students on their respective campuses alongside the combined challenges of a saturated market for mental health and wellness apps and a limited system infrastructure. In particular, both students and universities are interested in improving access, an increased focus on wellness, peer- community support, and culturally responsive mental health options. However, by not looking coherently and comprehensively across both the organization and the technologies that support them, most offerings fall short. Study findings provide insight for researchers, implications for designers and implementers of these systems, and hope for both students and therapists in developing a future with a comprehensive, aligned, and coherent user experience for mental health on campus.

\section{ACKNOWLEDGMENTS}

We would like to thank the Assistant Dean of Access and Inclusion at University of California Irvine, Dr. Sharnnia Artis, for assistance on this project.

\section{REFERENCES}

[1] Jacob Bor, Atheendar S Venkataramani, David R Williams, and Alexander C Tsai. 2018. Police killings and their spillover effects on the mental health of black Americans: a population-based, quasi-experimental study. The Lancet 392, 10144 (2018), 302-310.

[2] Rafael A. Calvo, Karthik Dinakar, Rosalind Picard, and Pattie Maes. 2016. Computing in Mental Health. Association for Computing Machinery 3438-3445 (2016), 8. https://doi.org/10.1145/2851581.2856463

[3] Cassandra Chaney and Ray V Robertson. 2013. Racism and police brutality in America. Fournal of African American Studies 17, 4 (2013), 480-505.

[4] Wang S.-w. Mancini C. McGrath-Mahrer B. Orama de Jesus S Chang, J. 2020. The complexity of cultural mismatch in higher education: Norms affecting firstgeneration college students' coping and help-seeking behaviors. Cultural Diversity and Ethnic Minority Psychology 26, 1 (2020), 280-294.

[5] Rosalie Corona, Vivian M Rodríguez, Shelby E McDonald, Efren Velazquez, Adriana Rodríguez, and Vanessa E Fuentes. 2017. Associations between cultural stressors, cultural values, and Latina/o college students' mental health. fournal of youth and adolescence 46, 1 (2017), 63-77.

[6] Patrick Corrigan. 2004. How stigma interferes with mental health care. American psychologist 59, 7 (2004), 614.

[7] Patrick W Corrigan, Benjamin G Druss, and Deborah A Perlick. 2014. The impact of mental illness stigma on seeking and participating in mental health care. Psychological Science in the Public Interest 15, 2 (2014), 37-70.

[8] Gavin Doherty, John Sharry, Magnus Bang, Mariano Alcañiz, and Rosa Baños. 2008. Technology in Mental Health. Association for Computing Machinery 39653968 (2008), 4.

[9] Deniece Dortch and Chirag Patel. 2017. Black undergraduate women and their sense of belonging in STEM at predominantly White institutions. NASPA fournal About Women in Higher Education 10, 2 (2017), 202-215.

[10] Andrea Gaggioli and Giuseppe Riva. 2013. From mobile mental health to mobile wellbeing: opportunities and challenges.. In MMVR. 141-147.

[11] Leslie RM Hausmann, Janet Ward Schofield, and Rochelle L Woods. 2007. Sense of belonging as a predictor of intentions to persist among African American and White first-year college students. Research in higher education 48, 7 (2007), 803-839.

[12] Marybeth Hoffman, Jayne Richmond, Jennifer Morrow, and Kandice Salomone. 2002. Investigating "sense of belonging" In first-year college students. Fournal of College Student Retention: Research, Theory \&Practice 4, 3 (2002), 227-256.

[13] Hugh Hunkin, Daniel L King, and Ian T Zajac. 2020. Perceived acceptability of wearable devices for the treatment of mental health problems. fournal of Clinical Psychology 76, 6 (2020), 987-1003.

[14] Justin Hunt and Daniel Eisenberg. 2010. Mental health problems and help-seeking behavior among college students. Journal of adolescent health 46, 1 (2010), 3-10.

[15] Gerald L Klerman. 1977. Mental illness, the medical model, and psychiatry. The journal of Medicine and Philosophy 2, 3 (1977), 220-243.

[16] Emily G Lattie, Rachel Kornfield, Kathryn E Ringland, Renwen Zhang, Nathan Winquist, and Madhu Reddy. 2020. Designing Mental Health Technologies that Support the Social Ecosystem of College Students. In Proceedings of the $2020 \mathrm{CHI}$ Conference on Human Factors in Computing Systems.1-15.

[17] Lipson-S. K., Eisenberg D, Lattie E. G. 2019. Technology and College Student Mental Health: Challenges and Opportunities. Frontiers In psychiatry 10, 1 (2019), 246.

[18] Seanna Leath and Tabbye Chavous. 2018. Black women's experiences of campus racial climate and stigma at predomi- nantly White institutions: Insights from a comparative and within-group approach for STEM and non-STEM majors. The Journal of Negro Education 87, 2 (2018), 125-139.

[19] Madhavan Mani, David J Kavanagh, Leanne Hides, and Stoyan R Stoyanov. 2015. Review and evaluation of mindfulness- based iPhone apps. FMIR mHealth and uHealth 3, 3 (2015), e82.

[20] Akihiko Masuda, Page L Anderson, and Joshua Edmonds. 2012. Help-seeking attitudes, mental health stigma, and self-concealment among African American college students. Fournal of Black Studies 43, 7 (2012), 773-786.

[21] Kelsey M Moffitt-Carney and Angela B Duncan. 2019. Evaluation of a mindfulnessbased mobile application with college students: A pilot study. fournal of American college health (2019), 1-7. 
[22] Brittany N Morey. 2018. Mechanisms by which anti-immigrant stigma exacerbates $\mathrm{racial} / \mathrm{ethnic}$ health disparities. American journal of public health 108, 4 (2018), 460-463.

[23] Samuel D Museus, Varaxy Yi, and Natasha Saelua. 2017. The impact of culturally engaging campus environments on sense of belonging. The Review of Higher Education 40, 2 (2017), 187-215.

[24] US News. 2020. The best national universities in America. https://www.usnews. com/best-colleges/rankings/national- universities (2020).

[25] Lydia O'donnell, Carl O'donnell, Dana Meritt Wardlaw, and Ann Stueve. 2004 Risk and resiliency factors influencing suicidality among urban African American and Latino youth. American journal of community psychology 33, 1-2 (2004), 37-49.

[26] Patrick O'Keeffe. 2013. A sense of belonging: Improving student retention. College Student fournal 47, 4 (2013), 605-613.

[27] Kathleen O'Leary, Arpita Bhattacharya, Sean A. Munson, Jacob O. Wobbrock, and Wanda Pratt. 2017. Design Opportunities for Mental Health Peer Support Technologies. Association for Computing Machinery 1470-1484 (2017), 15. https: //doi.org/10.1145/2998181.2998349

[28] Kathleen O'Leary, Stephen M. Schueller, Jacob O. Wobbrock, and Wanda Pratt 2018. "Suddenly, We Got to Become Therapists for Each Other": Designing Peer Support Chats for Mental Health. Association for Computing Machinery 1-14 (2018), 14. https://doi.org/10.1145/3173574.3173905

[29] Sara B Oswalt, Alyssa M Lederer, Kimberly Chestnut-Steich, Carol Day, Ashlee Halbritter, and Dugeidy Ortiz. 2020. Trends in college students' mental health diagnoses and utilization of services, 2009-2015. Fournal of American college health 68, 1 (2020), 41-51.

[30] N Eleni Pappamihiel and Marcio Moreno. 2011. Retaining Latino students: Culturally responsive instruction in colleges and universities. Fournal of Hispanic Higher Education 10, 4 (2011), 331-344.

[31] Monica Ruiz-Casares. 2014. Research ethics in global mental health: advancing culturally responsive mental health research. Transcultural psychiatry 51, 6 (2014), 790-805.

[32] Johnny Saldana. 2013. Qualitative data analysis: the coding Manual for qualitative researchers.
[33] Anneliese Singh.2020. Building a Counseling Psychology of Liberation: The Path Behind Us, Under Us, and Before Us. The Counseling Psychologist (2020), 0011000020959007.

[34] Lonnie Snowden. 2003. Bias in Mental Health Assessment and Intervention: Theory and Evidence. American fournal of Public Health 93, 1 (2003), 239-243.

[35] Katarzyna Stawarz, Chris Preist, and David Coyle. 2019. Use of smartphone apps, social media, and web-based resources to support mental health and well-being: Online survey. 7MIR mental health 6, 7 (2019), e12546.

[36] Stoyan R Stoyanov, Leanne Hides, David J Kavanagh, Oksana Zelenko, Dian Tjondronegoro, and Madhavan Mani. 2015. Mobile app rating scale: a new tool for assessing the quality of health mobile apps. 7MIR mHealth and uHealth 3, 1 (2015), e27.

[37] Terrell L Strayhorn. 2009. Fittin'in: Do diverse interactions with peers affect sense of belonging for Black men at predominantly White institutions? Fournal of Student Affairs Research and Practice 45, 4 (2009), 953-979.

[38] Terrell L Strayhorn. 2018. College students' sense of belonging: A key to educational success for all students. Routledge.

[39] Don Bambino Geno Tai, Aditya Shah, Chyke A Doubeni, Irene G Sia, and Mark L Wieland. 2020. The disproportionate impact of COVID-19 on racial and ethnic minorities in the United States. Clinical Infectious Diseases (2020).

[40] Janis Tondora, Maria O'Connell, Rebecca Miller, Tom Dinzeo, Chyrell Bellamy, Raquel Andres-Hyman, and Larry Davidson. 2010. A clinical trial of peer-based culturally responsive person-centered care for psychosis for African Americans and Latinos. Clinical Trials 7, 4 (2010), 368-379.

[41] John Torous, Keris Jän Myrick, Natali Rauseo-Ricupero ,and Joseph Firth. 2020. Digital mental health and COVID-19: Using technology today to accelerate the curve on access and quality tomorrow. FMIR mental health 7, 3 (2020), e18848.

[42] Marianne Webb, Jane Burns, and Philippa Collin. 2008. Providing online support for young people with mental health difficulties: challenges and opportunities explored. Early intervention in psychiatry 2, 2 (2008), 108-113.

[43] Lucretia Williams and Gloria Washington. 2018. Soul Glow: An Application for Helping to Understand and Improve Mental Health Care of HBCU Students. In 2018 IEEE International Conference on Healthcare Informatics (ICHI). IEEE, 358-360 